Authors' RePLy: In response to the letter from Dr Goldman, we should point out that our study demonstrated heterogeneity among polydipsic chronic psychiatric patients for the presence and subtype of SIADH. Of the twelve patients, three demonstrated no abnormality, seven demonstrated Type I SIADH and two demonstrated Type II SIADH. We explicitly stated that the differences between the findings of Goldman et al (1988) and our own might have resulted from a number of differences in experimental design, or from patient heterogeneity.

We believe that our assessment of the relationship between plasma AVP and serum osmolality is valid because the control group was assessed with the same assay under similar conditions. The control subjects were sitting where the blood was taken and the period of half an hour of sitting was chosen purposely to accommodate the fact that plasma AVP reaches a basal level by this time. There is no reason to suspect that normals studied in a similar 'naturalistic' design would not continue to exhibit a linear correlation between plasma vasopressin and plasma osmolality. Your correspondent notes that Allon et al (1990) have investigated the effects of smoking on renal concentrating ability, but the relevance of this study to Goldman's critique is doubtful as, in the Allon study, no measures of vasopressin were made. Our article reviewed the literature on the effects of smoking on vasopressin levels and we are not persuaded that smoking was the cause of the inappropriately high vasopressin levels in our patients.

Regarding the assessment of renal concentrating ability, we think that the similarity of the normal ranges of plasma AVP versus plasma osmolality in the two studies, both of which used radio-immunoassays for the measurement of AVP, justifies the use of the normal range of plasma AVP versus urine osmolality provided by Goldman et al. We agree that the pooling of urine over four-hour periods does somewhat reduce the precision of the estimates of urine osmolality, but the main subject of our investigation was vasopressin secretion rather than urine concentrating ability and our findings regarding the latter are consistent with decreased renal concentrating ability seen in normal subjects on prolonged high fluid intake. As we have mentioned, bladder dysfunction is common in these patients, and as a result an ideal method for the study of urine concentrating ability would have to employ bladder catheterisation. Unless this is done, any data concerning renal function must be interpreted with caution.

Emsley et al (1989) found that untreated psychotic patients without polydipsia dilute their urine less efficiently than normals after fluid loading; some patients had higher than normal baseline levels of vasopressin, and these patients showed the most pronounced antidiuretic states. The fact that nonpolydipsic, recently admitted, untreated psychotic patients have these abnormalities is not surprising. Whether chronically polydipsic pyschotic patients have an abnormality in urinary diluting capacity independent of abnormally high plasma levels of AVP remains moot.

N. J. Delva

J. S. LAWSON

Department of Psychiatry

Queen's University

Kingston, Ontario

Canada

S. L. LightMan

Charing Cross and Westminster Medical School

London, England

Institute of Psychiatry

J. L. Crammer

London, England

\section{Psychotic depression presenting as status epilepticus}

SIR: Griffiths \& Farmer (Journal, December 1990, 157, 909-91 i) reported a case of status epilepticus secondary to water intoxication, which in turn was secondary to a depressive psychosis. The patient admitted brief episodes of low mood over the previous ten years associated with an increase in fluid intake.

We were very interested to read this report since, as the authors state, there is a paucity of literature on water intoxication in subjects other than chronic schizophrenic patients. However, another perspective can be provided by a recent paper (Allon et al, 1990) reporting investigations of two schizophrenic patients admitted with hyponatremic seizures, and suggesting that cigarette use may contribute to the development of hyponatremia by impairing water excretion.

In their paper, Griffith \& Farmer did not mention whether the patient was a smoker, and reported that renal response to water loading was not assessed. It would be interesting to know whether the patient was a smoker, and if that was the case, if her consumption was greater during the depressive episodes. The higher prevalence of heavy smoking in chronic schizophrenic patients than in ambulatory depressive patients could perhaps explain the fact that water intoxication occurs predominantly in the former category.

The common point between schizophrenics and depressives, concerning tobacco, is perhaps the fact 
that they use it as an escape from boredom and as a stimulant.

Jean Claude Monfort

ANDRE Manus

Service de Psychiatrie

Hôpital Albert Chenevier

40 Rue de Mesly

94010 Creteil

France

\section{Reference}

Allon, M., Allen, H. M., Deck, L. V., et al. (1990) Role of cigarette use in hyponatremia in schizophrenic patients. American Journal of Psychiatry, 147, 1075-1077.

\section{Recording of information in case notes}

SIR: The medical defence societies regularly draw the attention of consultants to the proper recording of information in case notes, as it can have both clinical and medico-legal consequences. The fact that inadequacies continue, even in this increasingly litigious age, is regularly brought home to one of us (DD), in the capacity of second opinion appointed doctor and Mental Health Commissioner.

An audit of the use of lithium carbonate was conducted (by PLC) in a small district general hospital psychiatric unit. Of 73 patients on lithium treatment the case notes of 25 were randomly selected and studied in detail to investigate the adequacy of record-keeping and monitoring of treatment. In only two case notes was there any mention of information given about lithium treatment before it was started, and in no case notes was there any mention that the patient had been informed of the adverse and toxic effects, the need for monitoring, the need to maintain adequate fluid intake and so on.

It seems likely that if a similar survey was performed in most hospitals in this country, the same results would be obtained. Lithium is known to be a toxic drug, and patients are almost certainly counselled about its use, but there should be some mention of this in the case notes. If we fail to make notes in the case of such potentially dangerous drugs, we are much less likely to do so when the drug profile causes us less anxiety. If this was a routine practice some colleagues would have been able to avoid the heartache caused by the impending litigation over benzodiazepine dependence, where one of the most frequent allegations being made is that inadequate information was given about dependence and the need for gradual withdrawal.

Division of Psychiatry

The Royal Victoria Infirmary

Newcastle upon Tyne NE1 4LP

\section{Motor disorders of the mentally handicapped}

SIR: We agree with Rogers et al (Journal, January $1991,158,97-102$ ) that a crucial point in the motor disorders of the mentally handicapped population is to resolve the question of whether neuroleptic medication may contribute to motor disorders. We also agree that future studies on motor disorders of the mentally handicapped population should have a control group of similar population who have never been treated with neuroleptics.

Our pilot results from the dyskinesia screening project at the Cell Barnes Hospital (Kohen \& Mathew, Journal, 1990, 157, 621) show that $85.5 \%$ $(n=53)$ of the 62 randomly selected residents rate positively by a score of two in at least one area of the body as defined by DISCUS (Sprague et al, 1989). Of the 37 subjects who are on neuroleptic medication, $86.5 \%(n=32)$ and of the 25 subjects who have never been on neuroleptics, $84 \%(n=21)$ have a motor disorder in at least one area of the body.

The neuroleptic medication is clearly not a necessary or sufficient cause for the motor disorder in this complex and varied institutionalised population. The next step will be to delineate the anatomical distribution of the motor disorders in a significantly large population and to attempt to subtype the different subsyndromes according to the usage of neuroleptics, overlay of psychiatric illness, chronicity of the illness, length of stay in hospital and other demographic characteristics.

Charing Cross/Westminster Medical School

D. KOHEN

Unit of Mental Handicap

Fulham Palace Road

London W6 8RF

Cell Barnes Hospital

G. MATHEW

St Albans

Hertfordshire

\section{Reforence}

Sprague, R. L., Kalachnix, J. E. \& Shaw, K. H. (1989) Psychometric properties of the Dyskinesia Identification System; Condensed User Scale (DISCUS). Mental Retardation, 27, 141-148.

\section{Engagement of relatives in intervention programmes}

SIR: McCreadie et al (Journal, January 1991, 158, 110-113) report on their attempts to engage the relatives of schizophrenic patients in an intervention programme. They found a disappointingly high level of refusal by relatives-just over half of those approached. In our experience, the timing of offers of help is crucial; we approach relatives while the 